Pacific Journal of Mathematics

SOME MEASURE ALGEBRAS ON THE INTEGERS 


\section{SOME MEASURE ALGEBRAS ON THE INTEGERS}

\section{RICHARD SCOVILLE}

The author constructs some abstract algebras whose elements are subsets of the positive integers, and such that the measure of a set is its density. These algebras $\mathscr{A}$ are "abstract" in the sense that the countable join in the underlying lattice is not ordinary set union. However they are "concrete" in the sense that the elements of the algebra are sets, the notion of an integrable function is available and the normed vector space of integrable functions can be shown to be isometrically isomorphic to an ordinary $L^{1}$ space. If a function $f$ is integrable, it is shown that its integral is given by

$$
\lim _{N} \frac{1}{N} \sum_{j=1}^{N} f^{*}(j)
$$

where $f^{*}$ is a suitably chosen function differing from $f$ only on a set of density 0 .

This construction differs from others (several are described by Kubilius in his book on probabilistic methods in number theory), because usually countable additivity is sacrificed, whereas here the meaning of countable join has been altered.

The work was motivated by a desire to prove Theorems 3 and 4 which concern an application to sequences $(\bmod 1)$. We also include some remarks concerning the possibility of constructing probabilistically independent measure algebras. Furthermore by means of the Cantor expansion of a number we construct an algebra which contains all periodic sequences.

2. Construction of $\mathscr{A}$. Let $(X, \mathscr{B}, \mu)$ be a probability space. Let $\mathscr{F} \subseteq \mathscr{B}$ be an algebra (not necessarily a $\sigma$-algebra) which generates $\mathscr{B}$, i.e., $\mathscr{B}$ is the smallest $\sigma$-algebra containing $\mathscr{F}$. We refer to the members of $\mathscr{B}$ as Borel sets. Suppose also that a sequence $\left\{z_{n}\right\}$ of elements from $X$ is given satisfying

$$
\mu(I)=\lim \frac{1}{N} \operatorname{card}\left\{j \mid z_{j} \in I ; j=1,2, \cdots, N\right\} \text {, for all } I \in \mathscr{F} .
$$

This sequence will remain fixed throughout the discussion. A set $B \in \mathscr{B}$ is called admissible if there is a set of integers $A$ such that

$$
\mu(B \cap I)=\lim _{N} \frac{1}{N} \operatorname{card}\left\{j \mid z_{j} \in I ; j \in A ; j=1,2, \cdots, N\right\}, I \in \mathscr{F} .
$$


A will be said to go with $B$. A set of integers $A$ is said to have density if

$$
\lim _{N} \frac{1}{N} \operatorname{card}\{j \mid j \in A ; j=1,2, \cdots, N\}
$$

exists. The value of the limit will be written dens $(A)$. Moreover, if dens $\left(A_{1}-A_{2}\right)=0$ we will write $A_{1} \subseteq A_{2}$ (dens). We will write $A_{1}=A_{2}$ (dens) if $A_{1} \leqq A_{2}$ (dens) and $A_{2} \leqq A_{1}$ (dens). We state now a series of lemmas.

Lemma 1. If $A$ goes with $B$, then $\mu(B)=\operatorname{dens}(A)$.

Proof. $\mu(B)=\mu(B \cap X)=\operatorname{dens}(A)$.

Lemma 2. If $A$ goes with $B$ and if $A=A_{1}$ (dens), then $A_{1}$ goes with $B$.

Proof. Obvious.

LEMmA 3. If $I \in \mathscr{F}$, then $I$ is admissible and the set $\left\{j \mid z_{j} \in I\right\}$ goes with $I$.

Proof. Obvious.

Lemma 4. If $B$ is admissible, then $B^{c}$ is admissible.

Proof. If $A$ goes with $B, A^{c}$ goes with $B^{c}$.

Lemma 5. If $B_{1}$ and $B_{2}$ are admissible with $B_{1} \subseteq B_{2}$ and if $A_{1}$ and $A_{2}$ go with $B_{1}$ and $B_{2}$ respectively, then $A_{1} \subseteq A_{2}$ (dens).

Proof.

$$
\begin{aligned}
& \frac{1}{N} \operatorname{card}\left\{j \mid j \in A_{1}-A_{2}, j=1, \cdots, N\right\} \\
& =\frac{1}{N} \operatorname{card}\left\{j \mid j \in A_{1}-A_{2} ; z_{j} \in I ; j=1, \cdots, N\right\} \\
& +\frac{1}{N} \operatorname{card}\left\{j \mid j \in A_{1}-A_{2} ; z_{j} \notin I ; j=1, \cdots, N\right\} \\
& \leqq \frac{1}{N} \operatorname{card}\left\{j \mid j \in A_{2}^{c} ; z_{j} \in I ; j=1, \cdots, N\right\} \\
& +\frac{1}{N} \operatorname{card}\left\{j \mid j \in A_{1} ; z_{j} \notin I ; j=1,2, \cdots, N\right\} \text {. }
\end{aligned}
$$


Hence

$$
\begin{gathered}
\lim _{N} \frac{1}{N} \operatorname{card}\left\{j \mid j \in A_{1}-A_{2} ; j=1, \cdots, N\right\} \\
\leqq \mu\left(B_{2}^{c} \cap I\right)+\mu\left(B_{1} \cap I^{c}\right), I \in \mathscr{F} .
\end{gathered}
$$

Now since $\mathscr{F}$ generates $\mathscr{B}, \mu\left(I \triangle\left(B_{2}-B_{1}\right)\right)$ can be made as small as we wish so we see that

$$
\lim _{N} \frac{1}{N} \operatorname{card}\left\{j \mid j \in A_{1}-A_{2 \dot{m}} j=1, \cdots, N\right\}=0 .
$$

Lemma 6. If $A_{1}$ and $A_{2}$ go with $B$, then $A_{1}=A_{2}$ (dens).

Proof. This follows from Lemma 5.

LEMMA 7. If $B_{1}$ and $B_{2}$ are admissible with $B_{1} \cap B_{2}=\varnothing$ then we can find sets $A_{1}$ and $A_{2}$ going with $B_{1}$ and $B_{2}$, respectively, such that $A_{1} \cap A_{2}=\varnothing$.

Proof. Suppose $A_{1}$ and $A_{3}$ go with $B_{1}$ and $B_{2}$. Then $A_{3}^{c}$ goes with $B_{2}^{c}$, and since $B_{1} \subseteq B_{2}^{c}, A_{1} \subseteq A_{3}^{c}$ (dens). Hence $A_{3}-A_{1}=A_{3}$ (dens), so by Lemma 2 we may set $A_{2}=A_{3}-A_{1}$.

Lemma 8. If $B_{1} \subseteq B_{2}$ are both admissible then so is $B_{2}-B_{1}$.

Proof. Let $A_{1}$ and $A_{2}$ go with $B_{1}$ and $B_{2}$, respectively. By the preceding lemmas we can assume that $A_{1} \subseteq A_{2}$. Then for any $I \in \mathscr{F}$,

$$
\begin{aligned}
\mu\left(\left(B_{2}-\right.\right. & \left.\left.B_{1}\right) \cap I\right)=\mu\left(B_{2} \cap I\right)-\mu\left(B_{1} \cap I\right) \\
= & \lim _{N} \frac{1}{N} \operatorname{card}\left\{j \mid j \in A_{2} ; z_{j} \in I ; j=1, \cdots, N\right\} \\
& \quad-\lim _{N} \frac{1}{N} \operatorname{card}\left\{j \mid j \in A_{1} ; z_{j} \in I ; j=1, \cdots, N\right\} \\
= & \lim _{N} \frac{1}{N} \operatorname{card}\left\{j \mid j \in A_{2}-A_{1} ; z_{j} \in I ; j=1, \cdots, N\right\} .
\end{aligned}
$$

LEMmA 9. If $B_{1}, B_{2}, \cdots$, is a sequence of mutually disjoint admissible sets, then $\cup B_{i}$ is admissible.

Proof. Let $A_{1}, A_{2}, \cdots$, be a sequence of mutually disjoint sets going with $B_{1}, B_{2}, \cdots$, respectively. Since

$$
\lim _{N} \frac{1}{N} \operatorname{card}\left\{j \mid j \in A_{i} ; j=1, \cdots, N\right\}=\mu\left(B_{i}\right),
$$


we may assume, by removing a finite number of elements from $A_{i}$ if necessary, that for all $i$

$$
\frac{1}{N} \operatorname{card}\left\{j \mid j \in A_{i} ; j=1, \cdots, N\right\}<\mu\left(B_{i}\right)+2^{-i} \text { for all } N .
$$

Let $A=\cup A_{i}$ and let $I \in \mathscr{F}$. Then

$$
\begin{aligned}
& \frac{1}{N} \operatorname{card}\left\{j \mid z_{j} \in A ; j=1, \cdots, N\right\} \\
& \quad=\sum_{i=1}^{\infty} \frac{1}{N} \operatorname{card}\left\{j \mid z_{j} \in I ; j \in A_{i} ; j=1, \cdots, N\right\} .
\end{aligned}
$$

Now passage to the limit within the summation is justified since, by $(1)$, the series is dominated by the series $\sum\left(\mu\left(B_{i}\right)+2^{-i}\right)$. Hence we get

$$
\begin{gathered}
\lim _{N} \frac{1}{N} \operatorname{card}\left\{j \mid z_{j} \in I ; j \in A ; j=1, \cdots, N\right\} \\
=\sum \mu\left(B_{i} \cap I\right)=\mu(B \cap I) .
\end{gathered}
$$

LEMma 10. The admissible sets form a $\sigma$-algebra.

Proof. This is a simple consequence of Lemmas 8 and 9 .

THEOREM 1. Every Borel set is admissible.

Proof. This is obvious from Lemma 10 since the sets of $\mathscr{F}$ are admissible.

We denote by $\mathscr{A}$ the collection of all sets of integers which go with some $B \in \mathscr{B}$. Then it is clear from the preceding discussion that $\mathscr{A}$, modulo sets of density 0 , is isomorphic as a lattice to $\mathscr{B}$, modulo sets of measure 0 . We omit the details since the situation will be clearer after the definition of $L^{1}(\mathscr{A})$. However we will make one comment on the lattice operation in $\mathscr{A}$. For any sets $\left\{A_{i}\right\}$ from $\mathscr{A}$ going with sets $\left\{B_{i}\right\}$ from $\mathscr{B}$, let $\vee A_{i}$ be a set going with $\cup B_{i}$. Of course $\vee A_{i}$ is unique in $\mathscr{A}$ modulo sets of density 0 , but in addition the following is true.

THeOREM 2. Let $A_{1}, A_{2}, \cdots$, be sets from $\mathscr{A}$. Let $D$ be any set having density equal to dens $\left(\vee A_{i}\right)$ and suppose $D \supseteqq A_{i}$ (dens), $i=$ $1,2, \cdots$. Then $D=\vee A_{i}$ (dens).

Proof. Since $D-\vee A_{i} \subseteq D-\bigcup_{i=1}^{N} A_{i}$ (dens), and since

$$
\operatorname{dens}\left(D-\bigcup_{i=1}^{N} A_{1}\right)=\operatorname{dens}(D)-\operatorname{dens}\left(\bigcup_{i=1}^{N} A_{i}\right) \text {, }
$$


we see that $\operatorname{dens}\left(D-\vee A_{i}\right)=0$, i.e., $D \cong \vee A_{i}$ (dens). But since $\operatorname{dens}(D)=\operatorname{dens}\left(\vee A_{i}\right)$ we have also $\vee A_{i} \subseteq D$ (dens). Hence $D=$ $\vee A_{i}$ (dens).

This characterizes $\vee A_{i}$, modulo sets of density 0 , as the smallest set having a density and containing all the sets $A_{1}, A_{2}, \cdots$.

3. Applications to sequences $(\bmod 1)$. Let $T$ be the unit circle in the complex plane, and let $\left\{z_{n}\right\}$ be a sequence of points in $T$. We will say that $\left\{z_{n}\right\}$ has the distribution $\mu$ if $\mu$ is a probability measure on $T$ such that for any "interval," $I$, of $T$ (i.e., any connected subset of $T$ ) satisfying $\mu(\partial I)=0$, we have

$$
\mu(I)=\lim _{N} \frac{1}{N} \operatorname{card}\left\{j \mid z_{j} \in I ; j=1, \cdots, N\right\} .
$$

If we let $\mathscr{F}$ be the algebra of sets generated by those $I$ for which $\mu(\partial I)=0$, the preceding work is applicable and we get as special cases the following two theorems.

TheOREM 3. If $\left\{z_{n}\right\}$ has the distribution $\mu$, and if $\mu=\mu_{a t}+\mu_{c}$ where $\mu_{a t}$ is the atomic part of $\mu$, then there exists a set $A$ of integers, unique to within a set of density 0 such that, for any interval $I$ satisfying $\mu(\partial I)=0$,

$$
\mu_{a t}(I)=\lim _{N} \frac{1}{N} \operatorname{card}\left\{j \mid j \in A ; z_{j} \in I ; j=1, \cdots, N\right\} .
$$

THEOREM 4. If $\left\{z_{n}\right\}$ has the distribution $\mu$, and if $\mu=\mu_{1}+\mu_{2}$ where $\mu_{1}$ is absolutely continuous with respect to Lebesgue measure and $\mu_{2}$ is singular with respect to Lebesgue measure, there exists a set $A$ of integers, unique to within a set of density 0 such that

$$
\mu_{1}(I)=\lim _{N} \frac{1}{N} \text { card }\left\{j \mid j \in A ; z_{j} \in I ; j=1, \cdots, N\right\}
$$

and

$$
\mu_{2}(I)=\lim _{N} \frac{1}{N} \operatorname{card}\left\{j \mid j \in A^{c} ; z_{j} \in I, j=1, \cdots, N\right\}
$$

for any interval I for which $\partial I$ is $\mu$-null.

Proof. The proof uses only the fact that $\mu_{1} \perp \mu_{2}$ : there are disjoint Borel sets $B_{1}$ and $B_{2}$ such that $\mu_{1}(B)=\mu\left(B_{1} \cap B\right)$, and $\mu_{2}(B)=$ $\mu\left(B_{2} \cap B\right)$. Since the intervals having $\mu$-null boundaries generate $\mathscr{B}, B_{1}$ and $B_{2}$ are admissible. The conclusion of the theorem follows. 
4. Representation of $L^{1}(X)$ as a space of sequences. In this section we define a space $L^{1}(\mathscr{A})$ of sequences (actually equivalence classes of sequences) which is isometrically isomorphic to $L^{1}(X)$.

If $f$ is a sequence of real numbers, $f$ will be called measurable if for all real numbers $x$ in a dense set, we have

$$
D_{x}=\{j \mid f(j)<x\} \in \mathscr{A} \text {. }
$$

The function $\alpha(x)=$ dens $\left(D_{x}\right)$ is nondecreasing and of course can be extended to a nondecreasing function defined for all $x$. We will loosely refer to any such extension as the distribution of $f$. A measurable sequence $f$ will be called integrable if its distribution $\alpha$ satisfies

$$
\text { (i) } \int_{-\infty}^{\infty} d \alpha(x)=1 \text { and (ii) } \int_{-\infty}^{\infty}|x| d \alpha(x)<\infty \text {. }
$$

If $f$ is integrable, let $M(f)=\int_{-\infty}^{\infty} x d \alpha(x)$. Let $L$ be the set of all integrable sequences.

Lemma 11. If $f \in L$ has the distribution $\alpha$, then $D_{x} \in \mathscr{A}$ for every $x$ for which $\alpha$ is continuous.

Proof. We give only a sketch. Suppose $\alpha$ is continuous at $x_{0}$. Then for all $x, y$ with $x<x_{0}<y$ and $D_{x}, D_{y} \in \mathscr{A}$, we have $D_{x} \subseteq D_{x_{0}} \subseteq D_{y}$, so it is clear that dens $\left(D_{x_{0}}\right)$ exists. We must show that $D_{x_{0}}$ goes with a Borel set $B_{x_{0}}$. Since $D_{x}$ and $D_{y}$ are in $\mathscr{A}$, they go with Borel sets $B_{x}$ and $B_{y}$ with $B_{x} \subseteq B_{y}(\mu)$. Set $B_{x_{0}}=\cap B_{y_{i}}$, the intersection being taken over a decreasing sequence $\left\{y_{i}\right\}$ converging to $x_{0}$. Then it is easily verified that $D_{x_{0}}$ goes with $B_{x_{0}}$, proving the lemma.

We define a map $\phi$ from $L$ to $L^{1}(X)$ as follows: let $f \in L$ and construct a sequence of partitions $P_{n}=\left(\cdots, a_{-1, n}, a_{0, n}, a_{1, n}, \cdots\right)$ of the real line having the following properties:

(i) $\operatorname{mesh}\left(P_{n}\right)<2^{-n}(n=1,2, \cdots)$

(ii) $a_{2 i, n+1}=a_{i, n}(n=1,2, \cdots ; i=0, \pm 1, \pm 2, \cdots)$

(iii) $a_{i, n}$ is a point of continuity of $\alpha$.

Define

$$
D_{i, n}=\left\{j \mid a_{i, n} \leqq f(j)<a_{i+1, n}\right\}
$$

and let $B_{i, n}$ be a Borel set such that $D_{i, n}$ goes with $B_{i, n}$. Define, in $L^{1}(X)$,

$$
g_{n}=\sum_{i=-\infty}^{\infty} a_{i, n} \chi B_{i, n} .
$$

$\left\{g_{n}\right\}$ is clearly a Cauchy sequence in $L^{1}(X)$. Let $\phi(f)=\lim g_{n}$. The limit may be taken either a.e. or in $L^{1}(X)$. 
LEMma 12. $\phi(f)$ does not depend on the choice of the partitions $P_{n}$.

The proof is omitted. Suffice it to say that $\phi(f)$ could have been defined by a "spectral" integral of the form

$$
\phi(f)(\cdot)=\int_{-\infty}^{\infty} x d\left(\chi_{B_{x}}(\cdot)\right)
$$

where $D_{x}=\{j \mid f(j)<x\}$ goes with $B_{x}$.

TheOREM 5. If $f$ and $g$ are in $L$ and if $f$ has the distribution $\alpha$, then $\phi(f)=\phi(g)$ if and only if

$$
\{j \mid f(j)<x\}=\{j \mid g(j)<x\}(\text { dens })
$$

for every $x$ for which $\alpha$ is continuous.

Proof. Assume the condition is satisfied. If we choose partition points at which both $\alpha$ and $\beta$ (the distribution of $g$ ) are continuous, we get immediately $\phi(f)=\phi(g)$.

Conversely, suppose that $\phi(f)=\phi(g)$ and that $x$ is a point of continuity of both $\alpha$ and of $\beta$. Then as in the proof of Lemma 11 we see that both the sets $\{j \mid f(j)<x\}$ and $\{j \mid g(j)<x\}$ go with $\{z \mid \dot{\phi}(f)(z)<x\}$ and hence are equal (dens). It then follows that $\alpha=\beta$ on a dense set so the points of continuity of $\alpha$ are precisely the points of continuity of $\beta$. This proves the theorem.

We now set $f \equiv g$ if $\phi(f)=\phi(g)$, and let $[f]$ be the equivalence class containing $f$. The collection of all equivalence classes $[f](f \in L)$ will be denoted by $L^{1}(\mathscr{A})$.

Lemma 13. If $f \in L$ then there is a sequence $f^{*} \in L$ with $f=$ $f^{*}$ (dens) such that

$$
M(f)=\lim _{N} \frac{1}{N} \sum_{j=1}^{v} f^{*}(j)
$$

Here we have used the notation $f=g$ (dens) to mean that the set $\{j \mid f(j) \neq g(j)\}$ has density 0 . If $f=g$ (dens) then surely $f \equiv g$, but the converse need not be true as the example $f(j)=1 / j$ and $g(j)=0$ shows.

Proof. First assume that $\alpha$ is continuous at 0 . We can clearly treat the positive and negative parts of $f$ separately so we assume also that $f \geqq 0$. Let $0=\delta_{1}, \delta_{2}, \delta_{3}, \cdots$ be an increasing sequence of numbers at which $\alpha$ is continuous and such that $\lim \delta_{n}=\infty$. Let 


$$
f_{n}(j)= \begin{cases}f(j), & \delta_{n} \leqq f(j)<\delta_{n+1} \\ 0 & \text { otherwise }\end{cases}
$$

Since

$$
\lim _{N} \frac{1}{N} \sum_{j=1}^{N} f_{n}(j)=\int_{\delta_{n}}^{\delta_{n+1}} x d \alpha(x)
$$

we can modify $f_{n}$ on a set of density 0 (actually a finite set) so that for all $N$ the modified function $f_{n}^{*}$ satisfies

$$
\frac{1}{N} \sum_{j=1}^{N} f_{n}^{*}(j)<\int_{\delta_{n}}^{\delta_{n+1}} x d \alpha(x)+2^{-n}
$$

Let $f^{*}=\sum f_{n}^{*}$. Then

$$
\left\{j \mid f(j) \neq f^{*}(j)\right\} \subseteq\{j \mid f(j)>N\} \text { (dens) }
$$

for every $N$ so that $f=f^{*}$ (dens). Moreover

$$
\frac{1}{N} \sum_{j=1}^{N} f^{*}(j)=\sum_{n=1}^{\infty}\left(\frac{1}{N} \sum_{j=1}^{N} f_{n}^{*}(j)\right)
$$

Again the interchange of limits is justified by the dominated convergence, and we have

$$
\lim _{N} \frac{1}{N} \sum_{j=1}^{N} f^{*}(j)=\int_{0}^{\infty} x d \alpha(x)
$$

proving the lemma for those $f$ for which $\alpha$ is continuous at 0 . For any $f$ choose $x_{0}$ at which $\alpha$ is continuous. Consider $g=f-x_{0}$, apply the preceding to get $g^{*}$ and set $f^{*}=g^{*}+x_{0}$. Clearly $f^{*}$ has all the desired properties.

THEOREM 6. The space $L^{1}(\mathscr{A})$ is a vector space.

Proof. We must show that $L$ is closed with respect to addition and to multiplication by real numbers and that furthermore $f+g \equiv f^{\prime}+g^{\prime}$ and $c f \equiv c f^{\prime}$ whenever $f \equiv f^{\prime}$ and $g \equiv g^{\prime}$. Suppose $f$ and $g$ are in $L$. We must show that for all numbers $x$ in a dense set, $A_{x}=\{j \mid f(j)+$ $g(j)<x\} \in \mathscr{A}$. For each rational number $r_{i}$, let $A_{i}=\left\{j \mid f(j)<x-r_{i}\right.$; $\left.g(j)<r_{i}\right\}$. For any $M>0$ and $\varepsilon>0$, choose $N$ so large that a subcollection of the numbers $r_{1}, r_{2}, \cdots, r_{N}$ partitions the interval [ $\left.-M, M\right]$ with a mesh less than $\varepsilon$. Then for any $j \in A_{x-\varepsilon}-\bigcup_{i=1}^{N} A_{i}$ we have $f(j)+g(j)<x-\varepsilon$ and either $f(j)<x-r_{i}$ for all $r_{i} \in[-M, M], f(j) \geqq$ $x-r_{i}$ for all $r_{i} \in[-M, M]$ or for some $r_{i}, r_{j} \in[-M, M], f(j)<x-r_{i}$, $f(j) \geqq x-r_{j}$. In the first case $f(j)<x-M+\varepsilon$, in the second $f(j)>x+M-\varepsilon$, and in the third, since $j \notin A_{i}, g(j) \geqq r_{i}$. Since we 
may suppose $\left|r_{i}-r_{j}\right|<\varepsilon$ we get in the third case $f(j)+g(j) \geqq x-\varepsilon$. Hence the third case is impossible and we have

$$
A_{x-\varepsilon}-\bigcup_{i=1}^{N} A_{i} \subseteq\{j \mid f(j)>x+M-\varepsilon\} \cup\{j \mid f(j)<x-M+\varepsilon\} .
$$

Now since $\bigcup_{i=1}^{N} A_{i} \subseteq \vee A_{i}$ (dens), we see that $\operatorname{dens}\left(A_{x-\varepsilon}-\vee A_{i}\right)=0$, i.e., $A_{x-\varepsilon} \subseteq \vee A_{i}$ (dens). Let $T(x)=\operatorname{dens}\left(\vee A_{i}\right)$. Clearly $A_{x} \supseteqq A_{i}$ for all $i$, so we get for every $x$ and every $\varepsilon>0$,

$$
\underline{\operatorname{dens}}\left(A_{x}\right) \geqq \operatorname{dens}\left(\vee A_{i}\right)=T(x)
$$

and

$$
\overline{\operatorname{dens}}\left(A_{x-\varepsilon}\right) \leqq \operatorname{dens}\left(\vee A_{i}\right)=T(x) .
$$

Here we have used the notation dens and $\overline{\text { dens }}$ for lower and upper density respectively. Replacing $\bar{x}$ by $x+\varepsilon$ in the second inequality we get

$$
T(x) \leqq \underline{\operatorname{dens}}\left(A_{x}\right) \leqq \overline{\operatorname{dens}}\left(A_{x}\right) \leqq T(x+\varepsilon) .
$$

This shows that $A_{x}$ has a density if $x$ is a point of continuity of $T$ and Theorem 2 then gives $A_{x} \in \mathscr{A}$, since $A_{x}=\vee A_{i}$ (dens).

Note that for such $x$, the set $A_{x}$ goes with the Borel set $\{z \mid \phi(f)+\phi(g)<x\}(=\{z \mid \phi(f)+\phi(g) \leqq x\}(\mu))$. Hence $\phi(f+g)=\phi(f)+\phi(g)$. The only nontrivial point left is to prove that $-f \in L$ whenever $f \in L$. But $\{j \mid-f(j)<x\}=\{j \mid f(j)<-x\}=\{j \mid f(j) \leqq-x\}^{c}=\{j \mid f(j)<-x\}^{c} \in \mathscr{A}$ whenever $-x$ is a point of continuity of $\alpha$. This finishes the proof.

At the outset one is tempted to call a sequence $f$, "measurable," if for all $x,\{j \mid f(j)<x\} \in \mathscr{A}$. However, consider the following: let $D$ be a set not in $\mathscr{A}$ and define $f(j)=-1 / j$ and

$$
g(j)= \begin{cases}\frac{1}{j}, & j \notin D \\ \frac{1}{j}-\frac{1}{j^{2}}, & j \in D .\end{cases}
$$

Then $\{j \mid f(j)+g(j)<0\}=D \notin \mathscr{A}$, so that $f+g$ is not "measurable" even though $f$ and $g$ are.

THEOREM 7. If $L^{1}(\mathscr{A})$ is given the norm $|[f]|=M(|f|)$ then $\phi$ is an isometric isomorphism of $L^{1}(\mathscr{A})$ and $L^{1}(X)$.

Proof. It is clear from what has already been done that $\phi$ is an isometric isomorphism of $L^{1}(\mathscr{A})$ into $L^{1}(X)$. We must show that $\phi$ is onto, so let $\psi \in L^{1}(X)$ and suppose $\psi$ has distribution $\alpha, \alpha(x)=$ $\mu\{z \mid \psi(z)<x\}$. Choose partitions $P_{n}$ as before in the definition of $\phi$. 
For each $n$ let $A_{i, n}^{\prime}$ go with the Borel set $\left\{z \mid \psi(z)<a_{i, n}\right\}$. We can suppose that for all $i, A_{i, n}^{\prime} \subseteq A_{i+1, n}^{\prime}$ and that $A_{i, n+1}^{\prime} \subseteq A_{j, n}^{\prime}$ whenever $a_{i, n+1} \leqq a_{j, n}$. Set $A_{i, n}=A_{i+1, n}^{\prime}$ and define

$$
f_{n}(j)=\sum_{i} a_{i, n} \chi_{A_{i, n}}(j)
$$

and let $f(j)=\lim _{n} f_{n}(j)$. Now if $a_{k, n}<x<a_{k+1, n}$,

$$
A_{k, n}^{\prime}=\bigvee_{i=1}^{k-1} A_{i, n} \leqq\{q \mid f(q)<x\} \subseteq \bigvee_{i=1}^{k+1} A_{i, n}=A_{i+2, n}^{\prime} \text { (dens) . }
$$

This shows that if $x$ is a point of continuity of $\alpha$, the set $\{j \mid f(j)<x\}$ is in $\mathscr{A}$. This is what we wanted to prove.

5. Examples. In the first example we exhibit two algebras $\mathscr{A}_{1}$ and $\mathscr{C}_{2}$ that are independent; i.e., for any $A_{1} \in \mathscr{A}_{1}, A_{2} \in \mathscr{A}_{2}$, the set $A_{1} \cap A_{2}$ has density and dens $\left(A_{1} \cap A_{2}\right)=\operatorname{dens}\left(A_{1}\right)$ dens $\left(A_{2}\right)$. Let $T$ be the unit circle, let $X=T \times T$, let $\mu \times \mu$ be normalized Lebesgue (i.e., Haar) measure on $X$, let $\widetilde{\mathscr{B}}$ be the Borel sets of $X$, and let $\widetilde{F}$ be the algebra generated by the rectangles of $X$. Let $\{z, w) \in X$ be such that the sequence $\left\{(z, w)^{n}\right\}=\left\{\left(z^{n}, w^{n}\right)\right\}$ is uniformly distributed in $X$. Let $\mathscr{A}$ be the algebra determined by this sequence and let $\mathscr{A}_{1}$ and $\mathscr{A}_{2}$ be the algebras determined by $\left\{z^{n}\right\}$ and $\left\{w^{n}\right\}$, respectively. Let $A_{1} \in \mathscr{A}_{1}$ and $A_{2} \in \mathscr{A}_{2}$ go with Borel sets $B_{1}$ and $B_{2} \in \mathscr{B}$. We will show that, in $\mathscr{A}, A_{1}$ goes with $B_{1} \times T$ and $A_{2}$ goes with $T \times B_{2}$. Then it will follow that $A_{1} \cap A_{2}$ goes with $\left(B_{1} \times T\right) \cap\left(T \times B_{2}\right)=B_{1}$ so that dens $\left(A_{1} \cap A_{2}\right)=(\mu \times \mu)\left(B_{1} \times B_{2}\right)=\operatorname{dens}\left(A_{1}\right)$ dens $\left(A_{2}\right)$. But the fact that $A_{1}$ goes with $B_{1} \times T$ is clear: let $A_{1}^{\prime}$ go with $B_{1} \times T$ and consider $I \times T \in \mathscr{F}$. Then

$$
\begin{aligned}
\mu\left(B_{1} \cap I\right) & =(\mu \times \mu)\left(\left(B_{1} \times T\right) \cap(I \times T)\right) \\
& =\lim _{N} \frac{1}{N} \operatorname{card}\left\{j \mid(z, w)^{j} \in I \times T ; j \in A_{1}^{\prime} ; j=1, \cdots, N\right\} \\
& =\lim _{N} \frac{1}{N} \operatorname{card}\left\{j \mid z^{j} \in I ; j \in A_{1}^{\prime} ; j=1, \cdots, N\right\} .
\end{aligned}
$$

Hence $A_{1}^{\prime}=A_{1}$ (dens).

In this second example, we let $X=[0,1]$ and let $\mathscr{B}$ be the Borel sets of $X$. We are concerned with the sequence $\left\{z_{n}\right\}$ defined by

$$
z_{n}=\sum_{\jmath=2}^{\infty} \frac{n_{j}}{j !}
$$

where $n_{j}$ is the remainder obtained by dividing $n$ by $j, 0 \leqq n_{j}<j$.

Let $r$ be a rational number in $[0,1)$. We show that $\left\{z_{n}\right\}$ is uniformly distributed in $X$ by showing that 


$$
\lim _{N} \frac{1}{N} \operatorname{card}\left\{n \mid z_{n} \leqq r ; n=1, \cdots, N\right\}=r
$$

Let $k$ and $K$ be such that $r=k / K$ ! Note that $z_{n} \leqq k / K$ ! if and only if $n^{2} / 2 !+\cdots+n_{K} / K !<k / K !$, for suppose the latter. Then $n_{2} / 2 !+\cdots+n_{K} / K ! \leqq k-1 / K !$, so that $z_{n} \leqq k-1 / K !+\sum_{j=K+1}^{\infty} j-1 / j !=$ $k / K !$. The converse follows from the fact that for any $n, n_{j}=n$ for $j>n$. It follows that the set $\left\{n \mid z_{n} \leqq k / K !\right\}$ is periodic with period $K$ ! so its density is

$$
\frac{1}{K !} \operatorname{card}\left\{n \mid \frac{n_{2}}{2 !}+\cdots+\frac{n_{K}}{K !}<k / K ! ; n=0, \cdots, K !-1\right\}=k / K !=r .
$$

THEOREM 8. The algebra $\mathscr{C}$, constructed by means of the sequence $\left\{z_{n}\right\}$, contains all periodic sequences.

Proof. Let $K \geqq 2$ and let $p$ be between 0 and $K-1$. Let

$$
I=\bigcup_{i=0}^{(K-1) !-1}\left(\frac{i}{(K-1) !}+\frac{p}{K !}, \frac{i}{(K-1) !}+\frac{p+1}{K !}\right) .
$$

It is not difficult to see that $n_{K} \equiv\left[K ! z_{n}\right](\bmod K)$ so if $z_{n} \in I$ then $n_{K}=p$ and conversely. Hence $\left\{n \mid z_{n} \in I\right\}=\{n \mid n \equiv p(\bmod K)\}$. This proves the theorem.

Thanks are due to Professors L. Carlitz and O. Stackelberg with whom the author had many discussions during the preparation of this paper.

\section{REFERENCES}

1. N. Dunford and J. T. Schwartz, Linear operators, Part I, Interscience, New York, 1958.

2. J. Kubilius, Probabilistic methods in the theory of numbers, Translations of Mathematical Monographs, vol. 11, American Mathematical Society, Providence, R. I., 1964.

Received October 2, 1969.

DUKE UNIVERSITY

Durham, North Carolina 



\section{PACIFIC JOURNAL OF MATHEMATICS}

\section{EDITORS}

H. SAMELSON

Stanford University

Stanford, California 94305

\section{Richard Pierce}

University of Washington

Seattle, Washington 98105
J. DugundJI

Department of Mathematics

University of Southern California

Los Angeles, California 90007

RICHARD ARENS

University of California

Los Angeles, California 90024

\section{ASSOCIATE EDITORS}

\section{E. F. BeCKenBACH}

B. H. NeUmanN
K. YosHida

\section{SUPPORTING INSTITUTIONS}

\author{
UNIVERSITY OF BRITISH COLUMBIA \\ CALIFORNIA INSTITUTE OF TECHNOLOGY \\ UNIVERSITY OF CALIFORNIA \\ MONTANA STATE UNIVERSITY \\ UNIVERSITY OF NEVADA \\ NEW MEXICO STATE UNIVERSITY \\ OREGON STATE UNIVERSITY \\ UNIVERSITY OF OREGON \\ OSAKA UNIVERSITY \\ UNIVERSITY OF SOUTHERN CALIFORNIA
}

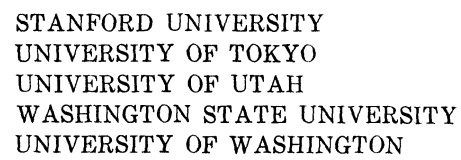

STANFORD UNIVERSITY UNIVERSITY OF TOKYO

UNIVERSITY OF UTAH

WASHINGTON STATE UNIVERSITY

UNIVERSITY OF WASHINGTON

The Supporting Institutions listed above contribute to the cost of publication of this Journal, but they are not owners or publishers and have no responsibility for its content or policies.

Mathematical papers intended for publication in the Pacific Journal of Mathematics should be in typed form or offset-reproduced, (not dittoed), double spaced with large margins. Underline Greek letters in red, German in green, and script in blue. The first paragraph or two must be capable of being used separately as a synopsis of the entire paper. The editorial "we" must not be used in the synopsis, and items of the bibliography should not be cited there unless absolutely necessary, in which case they must be identified by author and Journal, rather than by item number. Manuscripts, in duplicate if possible, may be sent to any one of the four editors. Please classify according to the scheme of Math. Rev. Index to Vol. 39. All other communications to the editors should be addressed to the managing editor, Richard Arens, University of California, Los Angeles, California, 90024.

50 reprints are provided free for each article; additional copies may be obtained at cost in multiples of 50 .

The Pacific Journal of Mathematics is published monthly. Effective with Volume 16 the price per volume (3 numbers) is $\$ 8.00$; single issues, $\$ 3.00$. Special price for current issues to individual faculty members of supporting institutions and to individual members of the American Mathematical Society: $\$ 4.00$ per volume; single issues $\$ 1.50$. Back numbers are available.

Subscriptions, orders for back numbers, and changes of address should be sent to Pacific Journal of Mathematics, 103 Highland Boulevard, Berkeley, California, 94708.

PUBLISHED BY PACIFIC JOURNAL OF MATHEMATICS, A NON-PROFIT CORPORATION

Printed at Kokusai Bunken Insatsusha (International Academic Printing Co., Ltd.), 7-17, Fujimi 2-chome, Chiyoda-ku, Tokyo, Japan. 


\section{Pacific Journal of Mathematics}

\section{Vol. 34, No. 3 \\ July, 1970}

Richard Hindman Bouldin, The peturbation of the singular spectrum

Hugh D. Brunk and Søren Glud Johansen, A generalized Radon-Nikodym derivative .

Henry Werner Davis, F. J. Murray and J. K. Weber, Families of $L_{p}$-spaces

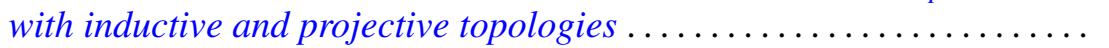

Esmond Ernest Devun, Special semigroups on the two-cell .

Murray Eisenberg and James Howard Hedlund, Expansive automorphisms

of Banach spaces ......................................

Frances F. Gulick, Actions of functions in Banach algebras.

Douglas Harris, Regular-closed spaces and proximities.

Norman Lloyd Johnson, Derivable semi-translation planes . .

Donald E. Knuth, Permutations, matrices, and generalized Young

tableaux..........................................

Herbert Frederick Kreimer, Jr., On the Galois theory of separable

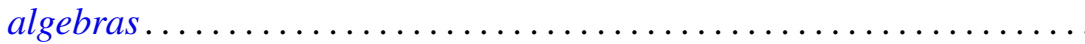

You-Feng Lin and David Alon Rose, Ascoli's theorem for spaces of

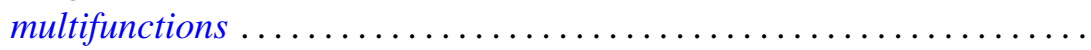

David London, Rearrangement inequalities involving convex functions . . . .

Louis Pigno, A multiplier theorem.

749

Helga Schirmer, Coincidences and fixed points of multifunctions into trees.

755

Richard A. Scoville, Some measure algebras on the integers .

Ralph Edwin Showalter, Local regularity of solutions of Sobolev-Galpern

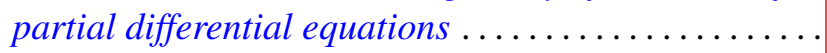

Allan John Sieradski, Twisted self-homotopy equivalences

John H. Smith, On S-units almost generated by S-units of subfields ...

803

Masamichi Takesaki, Algebraic equivalence of locally normal

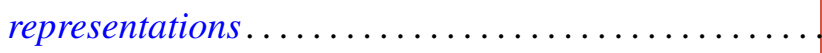

Joseph Earl Valentine, An analogue of Ptolemy's theorem and its converse in

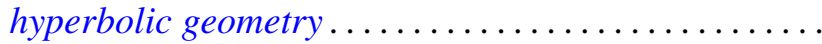

David Lawrence Winter, Solvability of certain p-solvable linear groups of finite order 